\title{
3 Research Square

\section{Quantitative Evaluation On Cardiac Remodeling Mechanism After Intensive Exercise: A Preliminary Study of Non-enhanced Cardiac Cine MRI}

hongqin Liang

First Affiliated Hospital to army medical University

Liqiang Zhu

The central theater command air force hospital of PLA

Bing Ji

First Affiliated Hospital to army medical University

Yongning Shang

First Affiliated Hospital to army medical University

xiaoyue Zhou

MR Collabration,Simens healthineers Ltd

Jian Wang ( $\sim 649672652 @ q q . c o m$ )

First Affiliated Hospital to army medicalUniversity

\section{Research Article}

Keywords: Cardiac MRI, Cardiac remodeling, Myocardial strain, Cardiac function, Exercise

Posted Date: July 30th, 2021

DOI: https://doi.org/10.21203/rs.3.rs-710342/v1

License: (c) (i) This work is licensed under a Creative Commons Attribution 4.0 International License.

Read Full License 


\section{Abstract}

Purpose: High intensity and longtime aerobic exercise may lead to the remodeling of both left and right ventricles with increased myocardial mass and cavity dilatation,which is mainly reflected in the changes of traditional cardiac function parameters.Feature tracking myocardial strain allows quantitative strain analysis of myocardial functionThe purpose was to quantitatively evaluate traditional cardiac function and feature tracking myocardial strain of exercise-induced, and

Materials and methods: The study included 67 healthy volunteers ( $21 \pm 2$ years of age). The exercise group $(n=43)$ who fulfilled our defined exercise criteria. The control group $(n=23)$ who maintained a basic daily life .Noncontrast enhancement CMR scanning were performed on all the subjects using a 3T MRI scanner .Cvi42 software was used for post-processing . Left ventricular cardiac function and overall globle stress were measured.

Results: Cardiac function parameters in the exercise group were significantly higher than those of the control group except for the ejection fractions (EFs) and heart rates (HRs). The GRS peak strain and GLS peak diastolic strain rates of both groups were significantly different $(P \otimes 0.05)$.The GRS peak strains and EFs were partly correlated $(\mathrm{R}=0.61)$. The GRS peak diastolic strains and cardiac Indices (Cls) were significantly correlated $(R=0.68)$. The GCS and GRS Peak Strains showed highly negative correlations $(R=-0.96)$. The GCS and GRS time to peak values were also highly correlated $(R=0.87)$.

Conclusion:The initial results showed that Changes in the functional parameters were more obvious than in the myocardial strain parameters, and some strain indices were correlated with the cardiac functional parameters, when the remodeling of the heart occurs. This is a new attempt to quantitatively assessment of Cardiac function and strain by Non-contrast-enhanced magnetic resonance.

\section{Introduction}

In 1899, the Swedish doctor, Hensehen [1] proposed the athlete heart concept. Since then, many scholars have confirmed that a certain amount of exercise can indeed cause changes, including the enlargement of cardiac chambers and alterations in cardiac function [2-4]. These exercise-induced adaptations are generally considered benign, but excessive exercise could lead to exercise-induced cardiac damage (exercise-induced myocardial ischemia, EIMI), and sudden cardiac death (SCD) [5-6], Thus, research on the mechanisms of cardiac remodeling in the early stage is needed.We need to assess whether the mechanisms of normal heart remodeling and pathological heart remodeling are consistent.

Traditionally, left ventricular remodeling has been primarily evaluated with ultrasound. However, due to the small field of vision and poor repeatability, left ventricular reconstructions were not as accurate as those of cardiovascular magnetic resonance (CMR)[7]. Recent studies suggest that CMR continues to have a unique advantage for detecting cardiac function , and assessing focal and diffuse myocardial fibrosis[8]. Late gadolinium enhancements (LGEs) of CMR imaging for detecting myocardial fibrosis have 
a distinct advantage over other methods; however, a recent study has shown that gadolinium might be deposited in the skin, dentate nucleus, and globus pallidus of patients with normal renal function[9] ..

Studies have shown that non-contrast-enhanced cardiac cine MRI was able to identify myocardial strain, a sensitive indicator of early cardiovascular dysfunction [10-11]. Non-contrast-enhanced cardiac cine MRI is a standard cardiac MRI examination method that can obtain end-diastolic volume index (EDVi) evaluations of left ventricular volume changes, myocardial mass index (Myo Mass) evaluations of overall quality changes, and mass-to-volume rate (MVR) evaluations of concentric circular remodeling of left ventricular myocardia[12]. Feature tracking (FT), which has been developed in recent years, tracks tissue motion between the inner and outer membrane boundaries of film sequences in the cardiac cycle. FT analyzes displacements and velocities for obtaining strains and strain rates. Based on longitudinal strain and the strain rates of central four-chamber films, radial and circumferential strain and strain rates were obtained for short-axis fillms[13]. The strain represents the rate of change in tissue lengths during myocardial movement:

$$
S t=\frac{L-L_{0}}{L_{0}}
$$

where $S_{t}$ is strain, $L_{0}$ is the initial length, and $L$ is the length at a certain point. Since the length of longitudinal and circumferential motion is shorter, longitudinal and circumferential strains are negative. Conversely, the thickness of the radial motion is increased, making radial strain positive. The immediate strain at the end of a contraction is usually recorded as the peak strain.

To this end, there are still no relevant researches about the relationship between cardiac function and strain after exercise using cardiac MRI films. In order to further explore the occurrence,outcome of the structure and function of the exercise heart, we aimed to investigate the relationship between cardiac functional and myocardial strain after intense exercises.

\section{Materials And Methods}

\section{Study Population}

72 participants were recruited.After excluding invalid information(( hypertension $(n=2)$, arrhythmia $(n=$ 1)), no heart-related diseases were found in rest of the volunteers during routine physical examinations before entering a group). 69 healthy volunteers were assessed. And all participants were divided into two groups( the exercise group and the control group).According to the criterias, the exercise group selfreported on the fulfillment of prespecified exercise criteria, as listed in the appendix(Table 1). The control group received daily basic life and exercise standards (using the exercise software, 5000-8000 steps/day only, and no weekly long-distance running, ball games, horizontal bar training, skipping rope, push-up, and sit-up training lasting for at least 4 years .All the recruited participants received CMR imaging. At last, the study included 67 healthy volunteers (the exercise group $(n=43)$ and the control group $(n=23))$ as showed in the flowchart (Fig. 1). 
Table 1

The exercise group received aerobic exercise of a certain intensity for 3-5times per week lasting for 4 years,Choose one of the sports standards between ball games and 3-5km long distance running. And the rest of the sports should be simultaneously satisfied by the gender

\section{Exercise Criteria}

Male

Female

horizontal bar training (30-50 times per day) sit-ups ( 50 times per day)

push-ups ( 50 times per day)

rope skipping ( $100-300$ times per day)

\section{Data collection}

The MRI scan was performed on all the subjects using a 3T MRI scanner (MAGNETOM Trio a Tim system, Siemens Healthcare, Erlangen, Germany) with a 6-channel body matrix coil plus 2 rows of the spine array coils, used to improve field uniformity with a target cardiac shimming mode. We compared the differences in the cardiac function parameters and myocardial strain values between the controls and exercise group. We acquired nonenhanced cardiac cine images with 25 reconstructed phases using balanced steady-state free-precession (bSSFP) sequence with retrospective electrocardiogram (ECG) gating. The collected subject information included the data from cardiac short axes, 2-chamber view, and 4-chamber view. The scan parameters were as the following: slice thickness $=6 \mathrm{~mm}$, time of echo $=1.7$ $\mathrm{ms}$, field of view $(F O V)=325 \times 400 \mathrm{~mm}^{2}$, matrix $=256 \times 256$, and slice thickness $=1.5 \mathrm{~mm}$. The commercial software, cvi42 (Circle Cardiovascular Imaging Inc., Calgary, Alberta, Canada), was used for post-processing using the 3D short module and 3D tissue tracking module. Endo- and epicardial contours were manually traced to measure the LV cavity at end-diastole and end-systole. The inner membrane contour was distanced from the blood pool, and the outer membrane contour was distanced from the fat outside of the heart and right ventricular blood pool to avoid the partial volume effects. The papillary muscles were included in the LV volume. The LV end-diastolic volume (EDV), end-systolic volume (ESV), stroke volume (SV), ejection fraction (EF), cardiac index (CI), myocardial mass index (Myo Mass ), global radial strain (GRS), global circumferential strain (GCS), and global longitudinal strain (GLS) parameters were obtained.

\section{Statistical Analyses}

The SPSS software version 19.0 (IBM Corp., Armonk/NY, USA) was used for data analysis. We compared the differences in each value using independent sample t tests. P-values $<0.05$ were considered statistically significant. The data were expressed as the means \pm the standard deviations. Mean differences between the groups were compared using paired t-tests and the Bland-Altman methods. The Pearson's method was used to analyze the relationships between the myocardial strain and cardiac function. The person that analyzed the MRIs was blinded to the study groups. For inter-observer 
reproducibility, two radiologists (each with $>10$ years of experience) independently analyzed the images from five randomly selected participants. Furthermore, one radiologist (with $>5$ years of experience) reanalyzed the images of ten participants after one month. The sample sizes were also calculated. Statistical tests were two-tailed, and the statistical significance was defined as a $p$-value $<0.05$. Strain measurements were very reproducible between the different examiners. Statistical analyses were performed using SPSS (version 21.0, SPSS, Inc., Chicago, IL, USA), GraphPad Prism (version 6.01, GraphPad Software, Inc., La Jolla, CA, USA), and MedCalc (version 11.4.2.0, MedCalc Software, Ostend, Belgium) software.

\section{Results}

Forty-three exercise enthusiasts (age: $20.67 \pm 1.08$ years; range: 18 to 24 years of age; $84 \%$ male) were enrolled who exercised 3-5 times per week for the past 4 years. Additionally, 23 control subjects with a similar age and sex distributions were studied (Table 2)

Table 2

The demographic parameters of all the participants

\begin{tabular}{|lll|}
\hline Parameters & Experimental group $(\mathbf{n}=\mathbf{4 3})$ & Control group $(\mathbf{n}=\mathbf{2 3})$ \\
\hline Age, years & $20.6 \pm 1.0$ & $21.3 \pm 2.3$ \\
\hline Weight, $\mathrm{kg}$ & $65.0 \pm 7.7$ & $62.0 \pm 8.3$ \\
\hline Height, $\mathrm{cm}$ & $173.6 \pm 6.0$ & $170.7 \pm 5.9$ \\
\hline Gender, male/female & $36 / 7$ & $16 / 7$ \\
\hline Body mass index, kg/m2 & $24.5 \pm 2.3$ & $23.2 \pm 1.9$ \\
\hline Body surface area, $\mathrm{m} 2$ & $1.8 \pm 0.2$ & $1.8 \pm 0.1$ \\
\hline Systolic blood pressure, $\mathrm{mmHg}$ & $110.0 \pm 2.1$ & $114.0 \pm 1.9$ \\
\hline Diastolic blood pressure, $\mathrm{mmHg}$ & $79.0 \pm 2.9$ & $81.0 \pm 2.1$ \\
\hline
\end{tabular}

A comparison of the cardiac function parameter results for all the participants is shown in Table 3. The left ventricular wall thickening rate and left ventricular motion were changed between the two groups(Figrue 2), and the cardiac function parameters of the exercise group were significantly higher than those of the control group, except for EF and HR, the differences in cardiac function parameters (EDV, ESV, SV, EF \%, Myo Mass \%) between the two groups were statistically significant $(p<0.05)$ (Figrue 3 ). 
Table 3

The cardiac function parameters between participants in the exercise and control groups.

\begin{tabular}{|llllc|}
\hline Parameters & Experimental group $(\mathbf{n}=\mathbf{4 3})$ & $\begin{array}{l}\text { Control group } \\
(\mathbf{n = 2 3})\end{array}$ & $\boldsymbol{t}$ & $\boldsymbol{P}$ \\
\hline $\mathrm{EDV} / \mathrm{ml}$ & $132.9 \pm 19.2$ & $104.9 \pm 15.2$ & 6.0 & $<0.01$ \\
\hline $\mathrm{ESV} / \mathrm{ml}$ & $53.4 \pm 10.0$ & $37.7 \pm 8.2$ & 6.4 & $<0.01$ \\
\hline $\mathrm{SV} / \mathrm{ml}$ & $79.4 \pm 13.0$ & $67.2 \pm 10.7$ & 3.8 & $<0.01$ \\
\hline $\mathrm{HR} /(\mathrm{BPM})$ & $65.1 \pm 7.3$ & $70.3 \pm 10.4$ & -2.3 & 0.02 \\
\hline $\mathrm{EF} / \%$ & $59.7 \pm 4.6$ & $64.1 \pm 5.5$ & -3.3 & $<0.01$ \\
\hline $\mathrm{CO} /\left(\mathrm{L} \cdot \mathrm{min}^{-1}\right)$ & $5.1 \pm 0.8$ & $4.7 \pm 0.8$ & 2.0 & 0.04 \\
\hline Myo Mass $(\mathrm{D}) / \mathrm{g}$ & $107.0 \pm 21.9$ & $73.2 \pm 16.2$ & 6.4 & $<0.01$ \\
\hline Myo Mass (S)/g & $106.5 \pm 22.1$ & $72.9 \pm 15.6$ & 6.4 & $<0.01$ \\
\hline $\begin{array}{l}\text { Data are displayed as median } \pm \text { SD. EDV End-Diastolic Volume; ESV End-Systolic Volume, SV Stroke } \\
\text { Volume ; HR Heart Rate; EF Ejection Fraction ; CO Cardiac Output; Myo Mass Myocardial Mass }\end{array}$ \\
\hline
\end{tabular}

The results comparing cardiac strain parameters in the participants are shown in Table 4. No significant differences were found in any strain parameters except for Global Radial Strain (GRS) peak strain and Global Longitudinal Strain (GLS) diastolic strain rates. The directional changes of strain in the heart caused by exercise, were primarily axial and longitudinal. The GRS peak strain and GLS peak diastolic strain rates were lower in the exercise group compared with the control group $(p<0.05)$, as shown in Fig. 4. 
Table 4

The cardiac strain parameters between the participants in the exercise and control groups

\begin{tabular}{|llllc|}
\hline Parameters & $\begin{array}{l}\text { Experimental group }(n= \\
\text { 43) }\end{array}$ & $\begin{array}{l}\text { Control group }(n= \\
\mathbf{2 3})\end{array}$ & $\boldsymbol{t}$ & $P$ \\
\hline GRS peak strain (\%) & $38.4 \pm 6.7$ & $43.9 \pm 11.2$ & -2.4 & 0.01 \\
$\begin{array}{l}\text { GRS peak systolic strain rate } \\
(1 / \mathrm{s})\end{array}$ & $2.8 \pm 3.3$ & $2.7 \pm 1.0$ & 0.1 & 0.89 \\
\hline $\begin{array}{l}\text { GRS peak diastolic strain rate } \\
(1 / \mathrm{s})\end{array}$ & $-2.9 \pm 0.8$ & $-3.3 \pm 1.2$ & 1.0 & 0.27 \\
\hline GRS peak displacement (mm) & $6.8 \pm 1.0$ & $6.4 \pm 0.7$ & 1.8 & 0.07 \\
\hline GCS peak strain (\%) & $-20.7 \pm 2.0$ & $-21.8 \pm 2.7$ & 1.9 & 0.06 \\
\hline $\begin{array}{l}\text { GCS peak systolic strain rate } \\
(1 / \mathrm{s})\end{array}$ & $-1.8 \pm 3.8$ & $-1.3 \pm 0.3$ & -0.6 & 0.05 \\
\hline $\begin{array}{l}\text { GCS peak diastolic strain rate } \\
(1 / \mathrm{s})\end{array}$ & $1.6 \pm 0.3$ & $1.6 \pm 0.4$ & 0.1 & 0.95 \\
\hline $\begin{array}{l}\text { GCS peak displacement (mm) } \\
\text { GLS peak strain (\%) }\end{array}$ & $2.8 \pm 11.3$ & $2.2 \pm 10.7$ & 0.2 & 0.84 \\
\hline $\begin{array}{l}\text { GLS peak systolic strain } \\
\text { rate(1/s) }\end{array}$ & $-19.0 \pm 2.8$ & $-19.3 \pm 2.3$ & 0.3 & 0.74 \\
\hline $\begin{array}{l}\text { GLS peak diastolic strain } \\
\text { rate(1/s) }\end{array}$ & $-0.9 \pm 1.4$ & $-1.1 \pm 0.2$ & 0.5 & 0.56 \\
\hline $\begin{array}{l}\text { GRS peak displacement (mm) } \\
\text { Data are displayed as median } \pm \text { SD. GCS, global circumferential strain; GLS, global longitudinal } \\
\text { strain;GRS global radial strain. }\end{array}$ & $4.3 \pm 3.0$ & $1.4 \pm 0.2$ & -2.9 & $<0.01$ \\
\hline
\end{tabular}

The correlations between cardiac function parameters and myocardial strain measurements are shown in Fig. 5. Significant correlations between GRS peak strains and EF percentages were found. The correlation coefficient between the GRS peak diastolic strain and the $\mathrm{Cl}$ was significant A highly negative correlation was found between the GCS and GRS Peak Strains, and the correlation coefficient of the GCS and GRS time-to-peak measurements was high..

\section{Discussion}

Exercise could cause changes in cardiac shapes, structures and functions. Some studies demonstrated that exercise can not only increase heart cavity volumes, but can also cause ventricular wall thickening 
and improve myocardial mass. Short exercise periods do not cause obvious changes in cardiac morphology. Cardiac enlargement is more pronounced as exercise training periods increase. This mechanical stimulus process is caused by preload and afterload pressures inducing myocardial hypertrophy[14].In this study, we assessed the feasibility of nonenhanced CMR imaging to detect myocardial strain and cardiac function after exercise. We demonstrated that 1) The ejection fractions and heart rate of participants in the exercise group were lower than those in the control group, 2) The functional parameter changes were more obvious than those of myocardial strain, and 3) Some myocardial strain index changes were correlated with cardiac function parameters.

The results of our study were consistent with previous research on the cardiac function. The cardiac function parameters of the exercise participants were higher than those of the control group, except for EFs and HRs. LVEFs are widely used and accurate for the evaluation of left ventricular systolic function. Ginzton et al[15] believed that during high-intensity exercise plays a major role in muscle contraction functions. We found that EFs of the exercise group were lower than those of the control group, suggesting that long-term high-intensity exercise resulted in marked depletion of left ventricular systolic function. These findings suggest that people who exercise with the intensity of those in our study should actually cut back on exercise regimens. As for decreased heart rates in the exercise group participants compared with the control group participants is consistent with previous research, which showed a reduction in the excitability of both the vagus and sympathetic nerve stimulations, however, sympathetic excitability was decreased more, causing reduced heart rates.

Previous studies have demonstrated that patients with Type 2 Diabetes Mellitus (T2DM) and hypertension have structural changes in the left ventricular myocardium and stress structures[16-18]. Myocardial strain, which reflects changes in myocardial mechanics, has been considered an important indicator of the myocardial deformation[19-20], and reductions in myocardial strain are found to obe earlier than those of EFs[21]. In our study, no significant statistical differences were found for all strain parameters, except for GRS peak strain and GLS diastolic strain rates, indicating that changes in strain directions occurred later than changes in cardiac function, inconsistent with previous research. This inconsistency might be because healthy enrolled volunteers had no pathologic cardiac changes, and the mechanisms behind cardiac changes were different, this might be the main difference between the normal heart and pathological heart. Cardiac muscle fatigue has been reported to be caused by extraordinary endurance exercises[22], however, recovery is quick after even very long events and does not appear to stimulate pathologic or biologic processes. These results stimulated our group to assess whether cardiac function parameters and myocardial strain would be reversible after the cessation of exercise and whether some association exist between the two. In addition, myocardial strain changes caused by exercise were primarily axial and longitudinal. The decrease in longitudinal and radial strain values in the exercise group indicated myocardial elasticity changes that could be responsible for the increased cardiac morphologies and decreased in EFs.

We also correlated cardiac function parameters with myocardial strain indicators. GRS peak strains and EFs were significantly correlated. GRS peak diastolic strain rates and Cls were also significantly 
correlated, suggesting that cardiac functional changes were closely related to radial strains of the myocardium during the early stage of remodeling. GCS and GRS Peak Strains were highly and negatively correlated, The GCS time-to-peak and GRS time-to-peak measurements were also highly and negatively correlated, suggesting that myocardial strain of both GRS and GCS was simultaneously affected and changed during cardiac remodeling.

There are some limitations which must be addressed. First, the sample size of this study was relatively small, and more volunteers should be included in future studies. Second, the volunteers will be recruited for longitudinal studies, although this was relatively complicated. The study results regarding the correlations between myocardial strain and cardiac function provided important findings for the study behind myocardial remodeling mechanisms. These results should be further verified and replicated by experiments with large sample sizes.

In summary, This study has several strengths. First, to the best of our knowledge, the cardiac magnetic resonance with its unique advantage has become the strong methods of evaluation on the heart disease,this is a new attempt to quantitatively assessment the effect of Cardiac change by Non-contrastenhanced magnetic resonance, which provides additional insight in the field of Cardiac remodeling. Second, this find out the change mechanism and potential correlation between heart function and myocardial strain in healthy heart, and the study of the mechanism of early changes in normal heart remodeling has opened up new noninvasive and reliable methods. Lastly, to find out the similarities and differences between normal and pathological heart change reporteded in recent literature., thereby expanding the scope of our findings.

\section{Abbreviations}

EIMI ( Exercise-Induced Myocardial Ischemia)

CMRI (Cardiovascular Magnetic Resonance Imaging)

GRS (Global Radial Strain)

GCS (Global Circumferential Strain)

GLS (Global Longitudinal Strain)

EF (Ejection Fraction)

HR (Heart Rate)

SCD (Sudden Cardiac Death)

LGE (Late Gadalinum Enhancement)

EDVi (End-Diastolic Volume index) 
MMi (Myocardial Mass Index)

MVR (Mass to Volume Rate)

FT (Feature Tracking)

T2DM (Type 2 Diabetes Mellitus)

\section{Declarations}

\section{Funding}

This work was supported by The Technology Innovation and Application Developmentof Chongqing city (No. cstc2019jscx-msxmX0126) and Chongqing Science and Health Joint Project $₫$ No.2021MSXM341).

\section{Ethics approval and consent participate}

This study was approved by the Ethics Committee at First Affiliated Hospital to Army Medical University (prot. KY2020083.May 20th, 2020) and designed in agreement with the 1964 Helsinki declaration and its later amendments. According to the Ethics Committee, all patients had to be informed about using their data for study purposes and give written consent before imaging examinations were allowed. The patients' information was anonymized prior to the analyses.

\section{Avalability of data material}

Data and material are available on request.

\section{Conflict of interest}

The authors declare that there was no conflict of interest concerning the conduct of the study or study outcomes.

\section{Authors' contributions}

Prof.WJ take responsibility for the conception and design of the study as the corresponding author. LQ designed the study, and wrote the manuscript. ZQ analyzed the datasets from the study and was in charge of statistics. They made equal contributions as the first author. JB was in charge of scaning , SN and ZY collected the datasets. All authors read and approved the final manuscript.

\section{Acknowledgement}

The authors would like to express their gratitude to the study participants for their involvement in the study and to reputable English language editing service (EditSprings)for help with English usage.

\section{References}


1. La Gerche A, Claessen G, Dymarkowski S, Voigt JU, De Buck F, Vanhees L, et al(2015)Exerciseinduced right ventricular dysfunction is associated with ventricular arrhythmias in endurance athletes. EurHeart J36:1998-2010.

2. Eijsvogels TMH, Oxborough DL, O'Hanlon R,Sanjay S, Sanjay P, Greg W, et al(2017)Global and regional cardiac function in lifelong endurance athletes with and without myocardial fibrosis. Eur $\mathrm{J}$ Sport Sci17:1297-1

3. Scally C, Rudd A, Mezincescu A, Wilson H, Srivanasan J, Horgan G, et al(2018)Persistent Long-Term Structural, Functional, and Metabolic Changes After Stress-Induced (Takotsubo) Cardiomyopathy.Circulation137:1039-10

4. Claus P, Omar AMS, Pedrizzetti G, Sengupta PP, Nagel E(2015)Tissue Tracking Technology for Assessing Cardiac Mechanics: Principles, Normal Values, and Clinical Applications. JACC Cardiovasc Imaging8:1444-1460. 5.Gati S, Sharma S, Pennell D(2018). The Role of Cardiovascular Magnetic Resonance Imaging in the Assessment of Highly Trained Athletes. JACC Cardiovasc Imaging112:247-259.

5. Levine BD(2014)Can intensive exercise harm the heart? The benefits of competitive endurance training for cardiovascular structure and function. Circulation 130:987-9

6. Turkbey EB, McClelland RL, Kronmal RA, Burke GL, Bild DE, Tracy RP, (2010)The impact of obesity on the left ventricle: the Multi-Ethnic Study of Atherosclerosis (MESA). JACC Cardiovasc Imaging3:266-2

7. Captur G, Muthurangu V, Cook C, Flett AS, Wilson R, Barison A, et al(2013)Quantification of left ventricular trabeculae using fractal analysis. J Cardiovasc Magn Reson15:36.

8. Raina S, Lensing SY, Nairooz RS, Pothineni NV, Hakeem A, Bhatti S, et al(2016)Prognostic Value of Late Gadolinium Enhancement CMRI in Systemic Amyloidosis. JACC Cardiovasc Imaging9:12671277.

9. Bohm P, Schneider G, Linneweber L, Rentzsch A, Krämer N, Abdul-Khaliq H, et al(2016)Right and Left Ventricular Function and Mass in Male Elite Master Athletes: A Controlled Contrast-Enhanced Cardiovascular Magnetic Resonance Study. Circulation133:1927-19

10. Situ YL, Birch SCM, Moreyra C, Holloway CJ(2020). Cardiovascular magnetic resonance imaging for structural heart disease.Cardiovasc Diagn Ther10:361-375.

11. Levelt E, Mahmod M, Piechnik SK, Ariga R, Francis JM, Rodgers CT, et al(2016)Relationship Between Left Ventricular Structural and Metabolic Remodeling in Type 2 Diabetes. Diabetes65:44-52.

12. Lang RM, Bierig M, Devereux RB, Flachskampf FA, Foster E, Pellikka PA, et al(2005)Recommendations for chamber quantification: a report from the American Society of Echocardiography's Guidelines and Standards Committee and the Chamber Quantification Writing Group, developed in conjunction with the European Association of Echocardiography, a branch of the European Society of Cardiology.J Am Soc Echocardiogr18:1440-1463.

13. Herm J, Töpper A, Wutzler A, Kunze C, Krüll M, Brechtel L, et al(2017)Frequency of exercise-induced ST-T-segment deviations and cardiac arrhythmias in recreational endurance athletes during a 
marathon race: results of the prospective observational Berlin Beat of Running study.BM J Open7(8): e015798

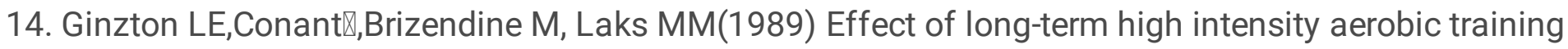
on left ventricular volume during maximal upright exercise.J Am Coll Cardiol 14:364-371.

15. Rider OJ, Lewandowski A, Nethononda R, Petersen SE, Francis JM, Pitcher A, et al(2013)Genderspecific differences in left ventricular remodelling in obesity: insights from cardiovascular magnetic resonance imaging. Eur Heart J34:292-299.

16. Hung CL, Verma A, Uno H, Shin SH, Bourgoun M, Hassanein AH,et al(2010)VALIANT investigators. Longitudinal and circumferential strain rate, left ventricular remodeling, and prognosis after myocardial infarction. J Am Coll Cardiol56:1812-1822.

17. Fontana M, White SK, Banypersad SM, Sado DM, Maestrini V, Flett AS, Piechnik SK, Neubauer S, Roberts N, Moon JC,et al(2012)Comparison of T1 mapping techniques for ECV quantification. Histological validation and reproducibility of ShMOLLI versus multibreath-hold T1 quantification equilibrium contrast CMRI. J Cardiovasc Magn Reson14:88.

18. Schuster A, Paul M, Bettencourt N, Morton G, Chiribiri A, Ishida M, et al(2013). Cardiovascular magnetic resonance myocardial feature tracking for quantitative viability assessment in ischemic cardiomyopathy.Int J Cardiol166:413-420.

19. Ide S, Riesenkampff E, Chiasson DA, Dipchand Al, Kantor PF, Chaturvedi RR, (2017)Histological validation of cardiovascular magnetic resonance T1 mapping markers of myocardial fibrosis in paediatric heart transplant recipients.J Cardiovasc Magn Reson19:10.

20. Tromp J, Lim SL, Tay WT, Teng TK, Chandramouli C, Ouwerkerk W, (2019)Microvascular Disease in Patients With Diabetes With Heart Failure and Reduced Ejection Versus Preserved Ejection Fraction. Diabetes Care42:1792-1799.

21. La Gerche A, Burns AT, Mooney DJ, Inder WJ, Taylor AJ, Bogaert J, (2012)Exercise-induced right ventricular dysfunction and structural remodelling in endurance athletes.Eur Heart J33:998-1006.

\section{Figures}


72 consecutive volunteers from June 1 to September 20,2020

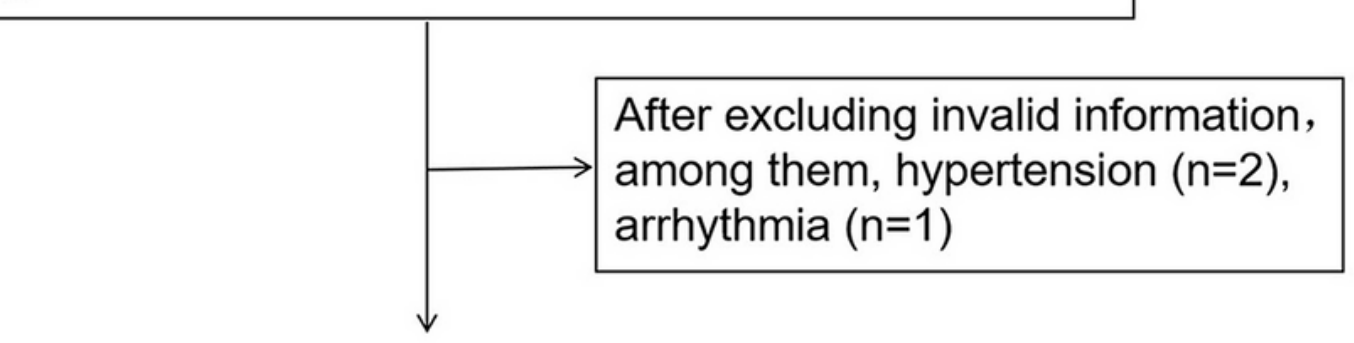

69 Healthy volunteers assessed

The exercise group self-reported on the fulfillment of prespecified exercise criter ia, as listed in the appendix. The control group received daily basic life and exer cise standards (using the exercise soft ware, $5000-8000$ steps/day lasting for a $\mathrm{t}$ least 4 years).
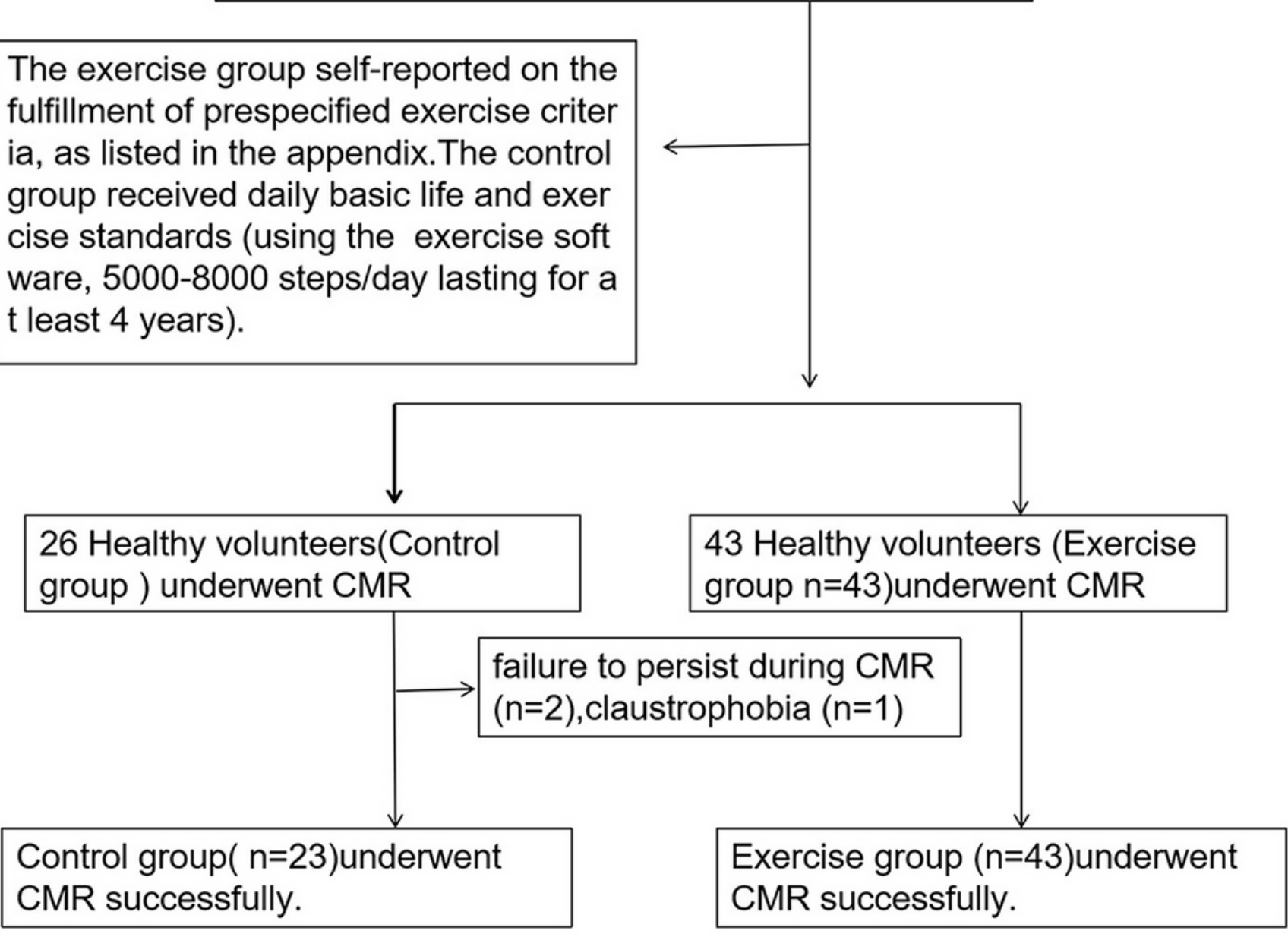

Figure 1

Flowchart of Subjects selection. 


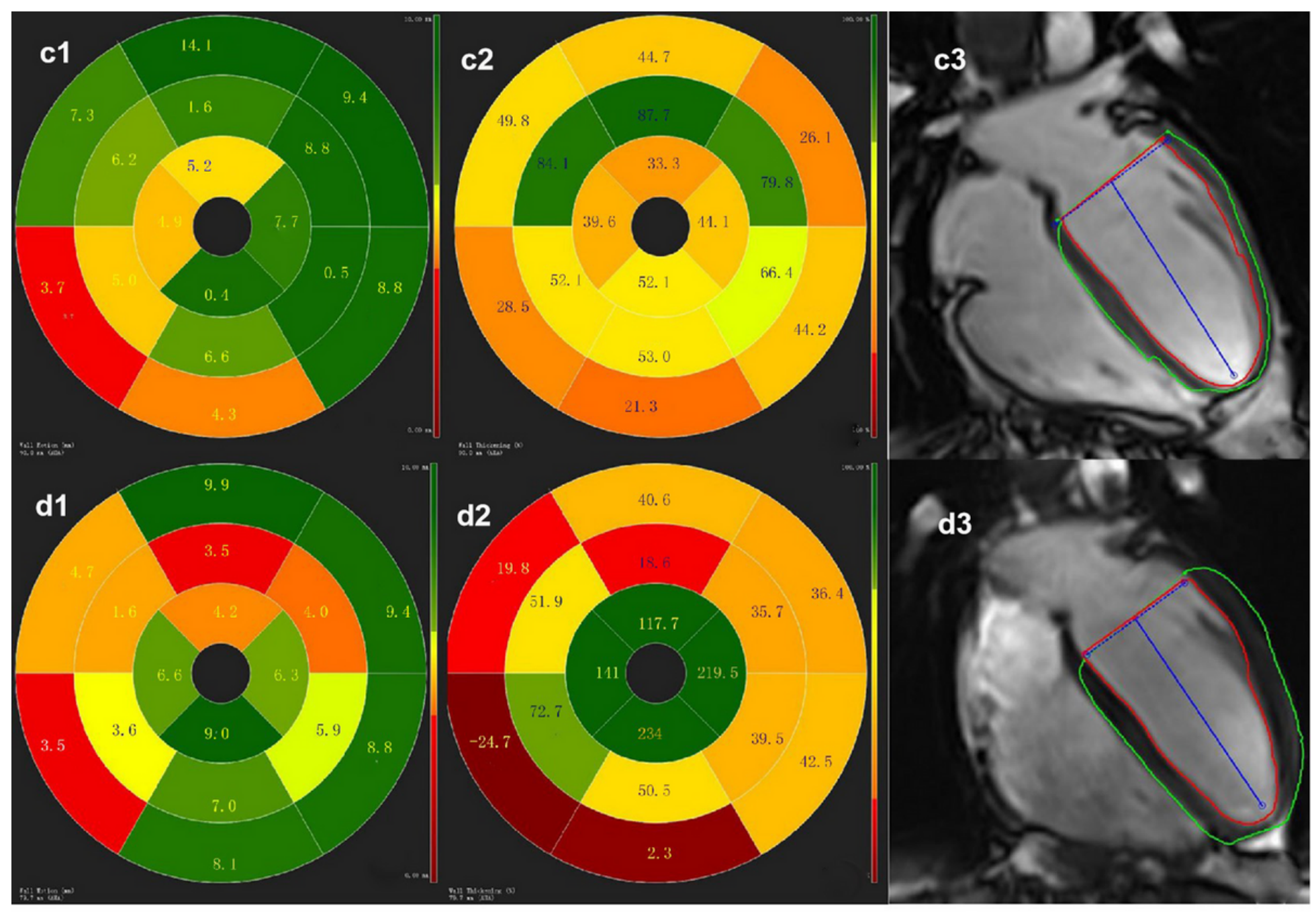

Figure 2

Compared with the control group(C1-C3), the left ventricular wall motion decreased, while the left ventricular wall thickening rate(\%) increased for exercise group(d1-d3) . 

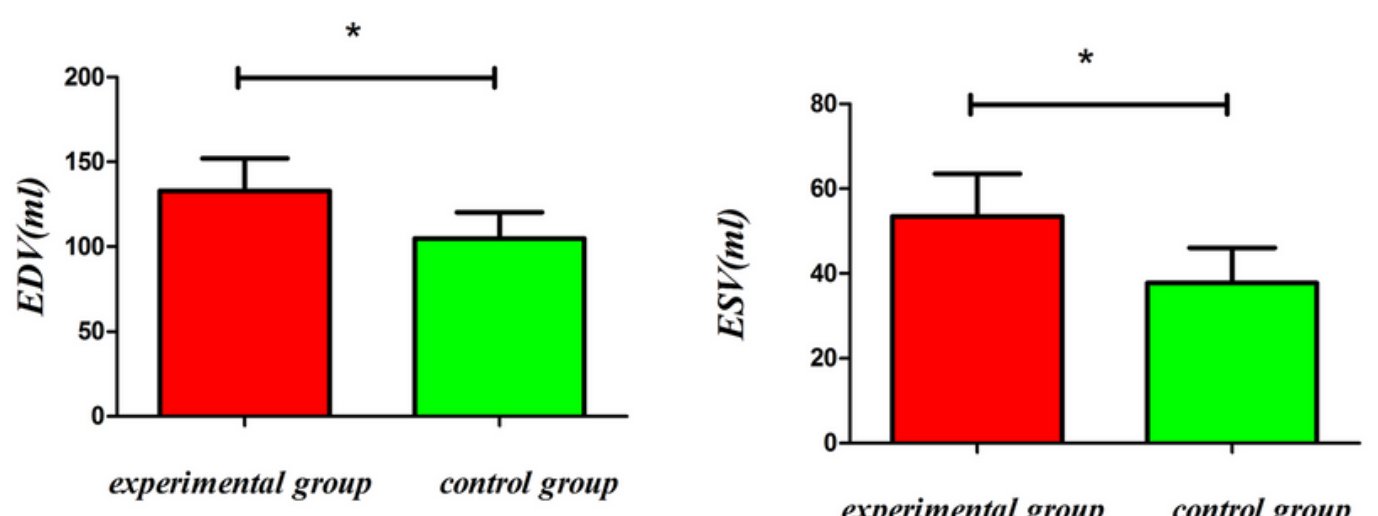

experimental group control group
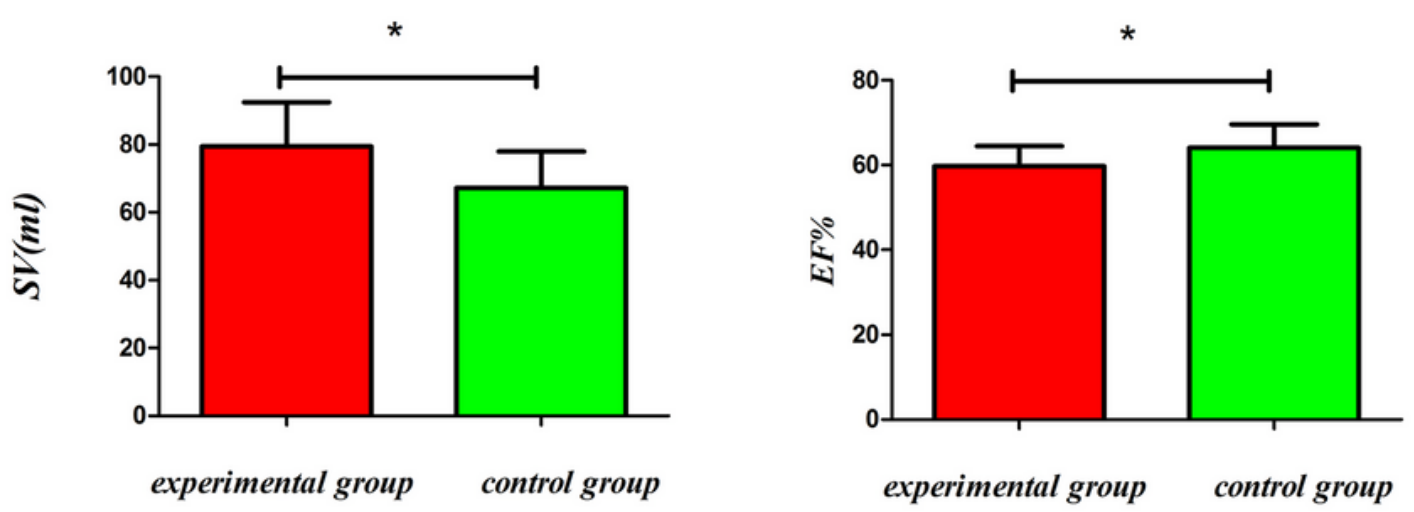

experimental group control group
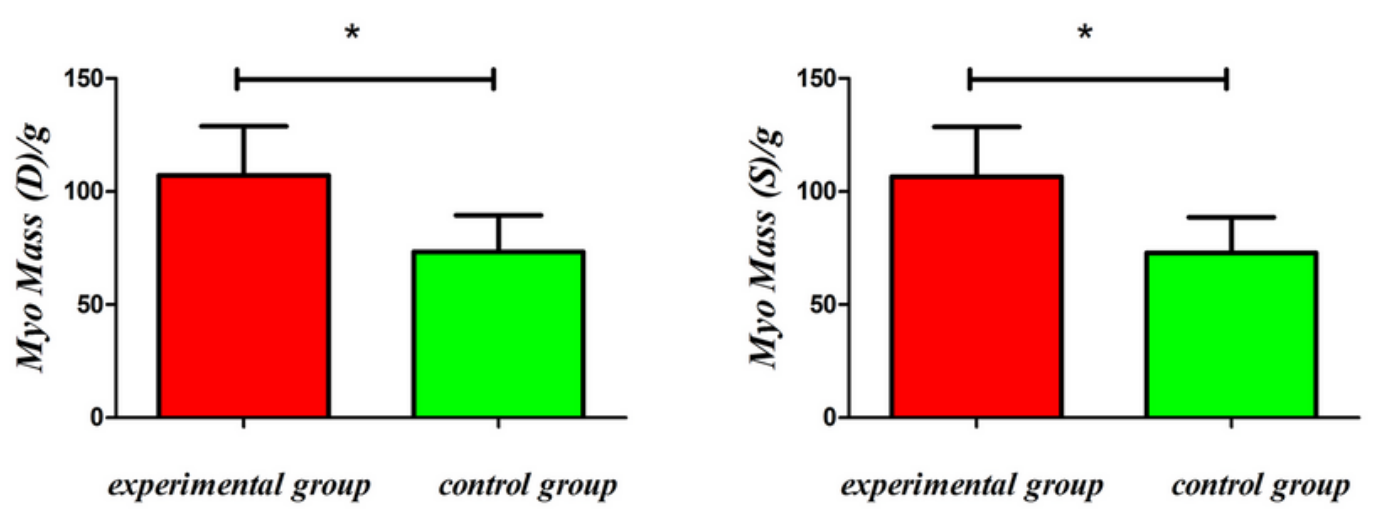

\section{Figure 3}

Bargraphs showing statistically significant differences between the exercise and control group for several cardiac function parameters. The end-diastolic volumes (EDVs) and end-systolic volumes (ESVs) were higher in the exercise than control group participants (TOP). The stroke volumes (SVs) were higher, and the ejection fraction percentages (EF\%) were lower in the Exercise than Control group participants (MIDDLE). The myocardial mass percentages in diastole (Myo Mass [D]\%) and systole (Myo Mass [S]\%) 
were higher in the exercise than the control group participants (BOTTOM). The means plus standard deviations are presented.

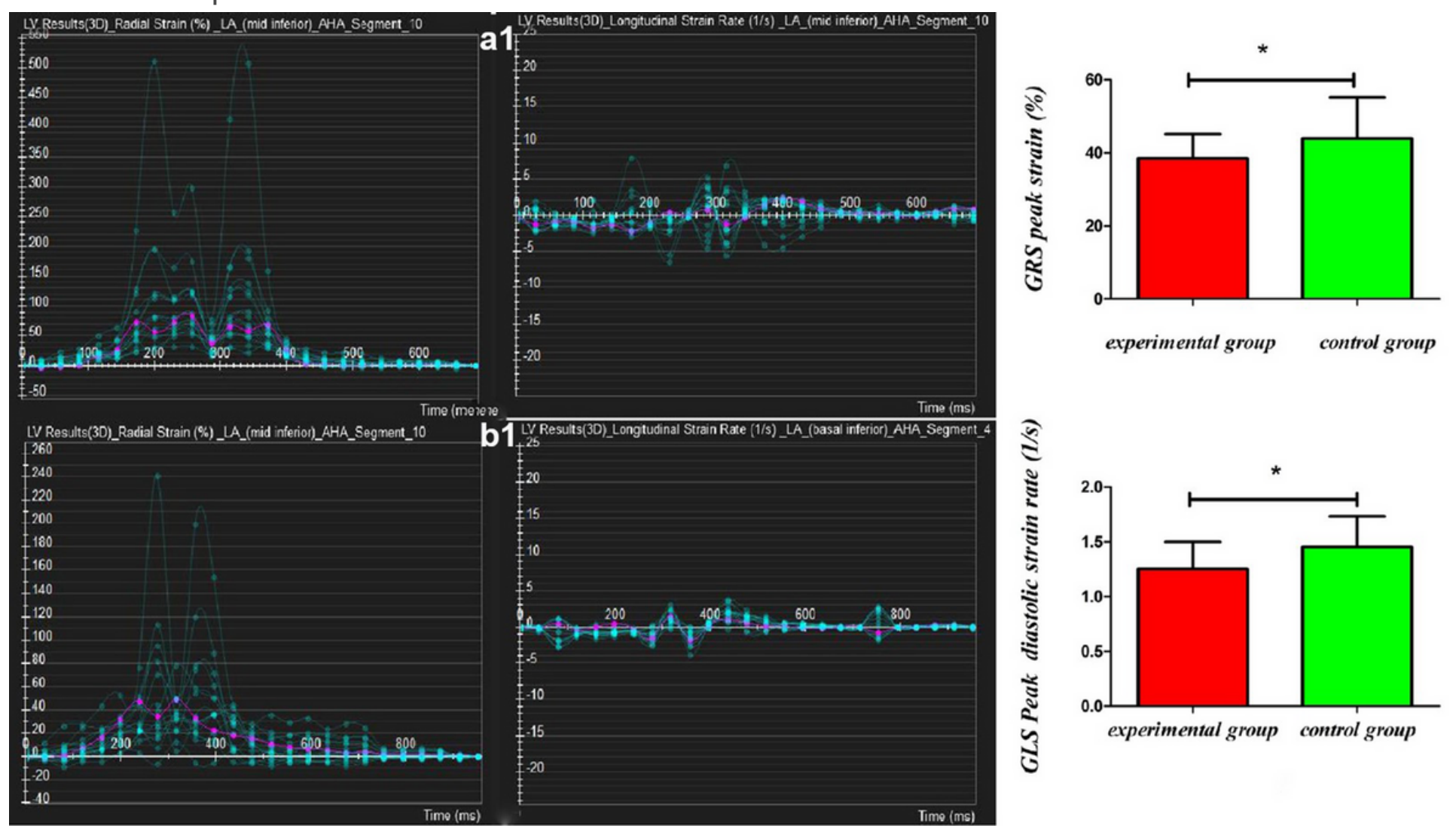

\section{Figure 4}

The strain degree and direction of longitudinal strain and axial strain were changed, compared with the control group,the strain degree of exercise group was increased and the myocardial synchronicity was decreased . 

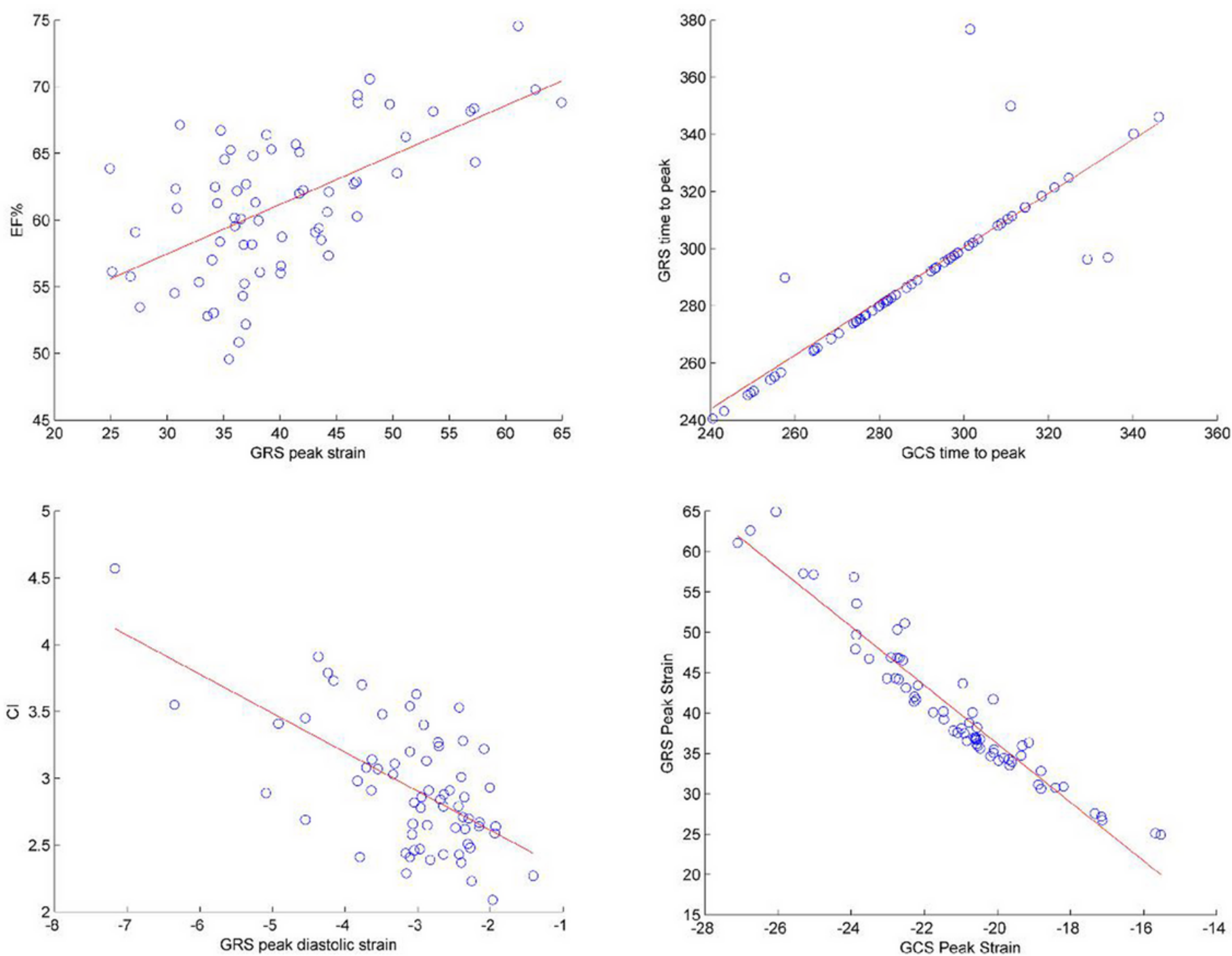

\section{Figure 5}

Correlation analyses of the cardiac function parameters and myocardial strain for all participants in the study. Global radial strain (GRS) peak strains and EF percentages were significantly correlated (TOP left; $\mathrm{R}=0.61, \mathrm{p} \otimes 0.05$ ). The correlation between the global circumferential strain (GCS) and GRS time-to-peak measurements was high (TOP right; $R=0.87$ ). The correlation between the cardiac index $(\mathrm{Cl})$ and $\mathrm{GRS}$ peak diastolic strain was negative and significant (BOTTOM left; $(R=-0.68, p \otimes 0.05)$. A strong and negative correlation was found between the GCS and GRS Peak Strains (BOTTOM right; R $=-0.96$ ). 